\title{
Atypical Chronic Myeloid Leukemia in a Patient with Aplastic Anemia
}

\author{
Michelina Santopietro ${ }^{a}$ Maurizio Miano ${ }^{b}$ Giovanna Palumbo ${ }^{a}$ Kejian Zhang ${ }^{c}$

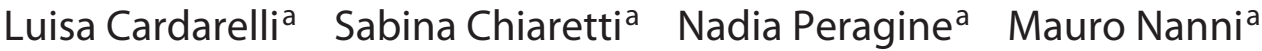 \\ Daniela Diverio $^{a}$ Francesca Mancini $^{a} \quad$ Anna Maria Testi ${ }^{a}$ Maria Luisa Moleti ${ }^{a}$ \\ Robin Foà $^{a}$ Fiorina Giona ${ }^{a}$ \\ ${ }^{a}$ Hematology, Department of Translational and Precision Medicine, Sapienza University of Rome, Rome, Italy; \\ ${ }^{b}$ Hematology Unit, IRCCS-Istituto Giannina Gaslini, Genoa, Italy; ' Division of Human Genetics, Cincinnati Children's \\ Hospital Medical Center, Cincinnati, OH, USA
}

Acquired aplastic anemia (AA), a bone marrow (BM) failure disease, and atypical chronic myeloid leukemia (aCML), a myelodysplastic syndrome (MDS)/myeloproliferative neoplasm, are both rare diseases. An aCML occurring as a secondary malignancy is a very rare event.

In April 2005, an 18-year-old boy already diagnosed with AA and treated with cyclosporine (CSA) and prednisone (PDN) came to our center. No HLA identical family donor was available. Cytogenetic analysis showed a normal karyotype. Treatment with rabbit anti-thymocyte globulin combined with CSA, methylprednisolone, and granulocyte colony stimulating factor was successfully administered. Forty-four months later, an asymptomatic severe thrombocytopenia was successfully treated with CSA and PDN. The peripheral blood cell count remained normal for 8 years, when a mild thrombocytopenia occurred. Cytogenetic analysis showed 46,XY, del(3) (p13p23)[20]. Nine months later, an increase in white blood cell count with $27 \%$ immature dysplastic myeloid cells (promyelocytes, myelocytes, and metamyelocytes) combined with thrombocytopenia and mild anemia was detected. No organomegaly was present. BM hyperplasia with granulocytic dysplasia and no fibrosis were present.
No BCR-ABL1, PDGFRA, PDGFRB, or FGFR1 rearrangements and no JAK2, CALR, CSF3R, SETBP1, TERC, or TINF2 mutations were detected. A diagnosis of aCML was made and hydroxycarbamide was started but the clinical and hematologic features worsened. While waiting for a haploidentical stem cell transplant, ponatinib (Ariad Pharmaceuticals Inc., Italy) was administered, based on in vitro sensitivity. Due to disease progression, ponatinib was discontinued and cytarabine, vindesine, and etoposide were given. Unfortunately, the patient developed an acute myeloid leukemia (AML) and died from multi-organ failure. The patient's DNA was then analyzed for genes associated with BM failure, AML, and MDS. Sanger sequencing defined two heterozygous missense mutations in the second zinc finger domain of the GATA2 gene (p.Thr358Lys and p.Leu359Val).

To date, there are no reported cases of AA evolving into aCML with clonal abnormalities involving chromosome 3. In our patient, the del(3)(p13p23), detected before the onset of aCML, could have been a prodromal sign of clonal evolution.

Cytogenetic abnormalities found in myeloid malignancies have been reported in $4-11 \%$ and $20-88 \%$ of AA and aCML, respectively [1-3]. To date, no association be-

\section{KARGER}

(c) 2019 S. Karger AG, Basel

E-Mail karger@karger.com

www.karger.com/aha
Michelina Santopietro, MD

Hematology, Department of Translational and Precision Medicine, Sapienza University of Rome/Azienda Ospedaliero-Universitaria Policlinico Umberto I Via Benevento 6, IT-00161 Rome (Italy)

E-Mail santopietro@bce.uniroma1.it 
tween hematologic diseases and del(3)(p13p23) has been reported. Interestingly, a deletion of the short arm of chromosome 3 revealed by cytogenetics does not correlate with GATA2 mutation involving the long arm of chromosome 3. GATA2 germ-line mutations are associated with the "GATA2 deficiency syndrome" and have also been identified in chronic neutropenia, in pediatric $\mathrm{BM}$ failure, and in young adults with AA. Acquired mutations of GATA2 are reported in MDS/AML [4] and the GATA2 L359V mutation has been found in CML patients in blast crisis [5]. Unfortunately, due to a lack of biologic samples, we were unable to define whether GATA2 T358L and $\mathrm{L} 359 \mathrm{~V}$ mutations, detected in our patient, were germline or acquired. As the patient had never suffered from monocytopenia and infections, we can only hypothesize that the GATA2 mutations were acquired. The results, hereby reported, suggest that GATA2 mutations should be investigated in all patients with AA.

\section{Statement of Ethics}

Informed consent to use the presented data anonymously was given by the patient.

\section{Disclosure Statement}

The authors declare no conflicts of interest.

\section{Funding Sources}

This research did not receive any specific grant from funding agencies in the public, commercial, or not-for-profit sectors.

\section{References}

1 Socié G, Rosenfeld S, Frickhofen N, Gluckman E, Tichelli A. Late clonal diseases of treated aplastic anemia. Semin Hematol. 2000 Jan;37(1):91-101.

2 Breccia M, Biondo F, Latagliata R, Carmosino I, Mandelli F, Alimena G. Identification of risk factors in atypical chronic myeloid leukemia. Haematologica. 2006 Nov;91(11):1566-

3 Hernández JM, del Cañizo MC, Cuneo A, García JL, Gutiérrez NC, González M, et al Clinical, hematological and cytogenetic char- acteristics of atypical chronic myeloid leukemia. Ann Oncol. 2000 Apr;11(4):441-4

4 Shiba N, Funato M, Ohki K, Park MJ, Mizushima Y, Adachi S, et al. Mutations of the GATA2 and CEBPA genes in paediatric acute myeloid leukaemia. Br J Haematol. 2014 Jan;164(1):142-5.

5 Zhang SJ, Ma LY, Huang QH, Li G, Gu BW, Gao XD, et al. Gain-of-function mutation of GATA-2 in acute myeloid transformation of chronic myeloid leukemia. Proc Natl Acad Sci USA. 2008 Feb;105(6):2076-81. 